\title{
Tracking the moving optical photocenters of active galaxies: binary black holes, accretion disks, and relativistic jets
}

Ann E. Wehrle, Stephen C. Unwin, Dayton L. Jones, David L. Meier, B. Glenn Piner

Ann E. Wehrle, Stephen C. Unwin, Dayton L. Jones, David L. Meier, B. Glenn Piner, "Tracking the moving optical photocenters of active galaxies: binary black holes, accretion disks, and relativistic jets," Proc. SPIE 4852, Interferometry in Space, (26 February 2003); doi: 10.1117/12.460869

SPIE Event: Astronomical Telescopes and Instrumentation, 2002, Waikoloa, Hawai'i, United States 


\title{
Tracking the Moving Optical Photocenters of Active Galaxies: Binary Black Holes, Accretion Disks and Relativistic jets
}

\author{
A. Wehrle ${ }^{a}$, S. C. Unwin ${ }^{b}$, D. L. Jones ${ }^{b}$, D. L. Meier ${ }^{b}$, and B. G. Piner ${ }^{c}$ \\ ${ }^{a}$ Interferometry Science Center, California Institute of Technology; \\ Mailing address: Jet Propulsion Laboratory, California Institute of Technology, \\ Mail Stop 301-486, 4800 Oak Grove Drive, Pasadena, CA 91109, U.S.A. \\ ${ }^{b}$ Jet Propulsion Laboratory, California Institute of Technology, \\ Mail Stop 301-486, 4800 Oak Grove Drive, Pasadena, CA 91109, U.S.A. \\ ${ }^{c}$ Whittier College, Department of Physics and Astronomy, \\ 13406 E. Philadelphia Street, Whittier, CA 90608
}

\begin{abstract}
We will use the astrometric capabilities of the Space Interferometry Mission to answer three key questions about active galacitic nuclei: 1) Does the separation of the radio core and optical photocenter of quasars change on the same timescale as their photometric variability, or is the separation stable? 2) Does the most compact optical emission from an active galactic nucleus come from an accretion disk or from a relativistic jet? 3) Do the cores of galaxies harbor binary supermassive black holes remaining from galaxy mergers? We will compare the radio and optical positions of quasars used in the tie between optical and radio celestial reference frames. During the first year after launch, we will be able to show whether the frame tie will be limited by "astrophysical noise".
\end{abstract}

Keywords: Space Interferometry Mission, SIM, Quasar, Astrometry, Active Galactic Nucleus

\section{INTRODUCTION}

Supermassive black holes in the centers of elliptical galaxies sporadically give rise to relativistic jets. Conditions may be briefly favorable to jet formation after two galaxies collide and merge. In some AGN, for example, NGC 4261, a few-hundred-pc-scale "dusty torus" is seen orbiting the black hole system, as observed by Ferrarese et al. ${ }^{1}$ and Jaffe et al. ${ }^{2}$ using the Hubble Space Telescope. This torus surrounds and acts as a fuel reservoir to an inner accretion disk- black hole system which, in turn, forms the true powerhouse of the AGN. In radio-loud AGN, two oppositely-directed radio jets are ejected perpendicular to the torus/disk system. In a few cases, we have been able to determine the intrinsic speed of the jet: for example, in $3 \mathrm{C} 279$, the particles in the jet move at 0.997 times the speed of light. What we do not understand is how the black hole- accretion disk system produces the jets, nor what structures exist within one light-year of the supermassive black hole.

Our SIM observations address three key questions about AGN:

1. Does the most compact optical emission from an AGN originate in an accretion disk or in a relativistic jet?

2. Does the separation of the radio core and optical photocenter of the most compact radio-loud quasars change on the timescales of their photometric variability, or is the separation stable?

3. Do the cores of galaxies harbor binary supermassive black holes remaining from galaxy mergers?

Further author information: Send correspondence to A.W.; E-mail: ann.wehrle@jpl.nasa.gov 


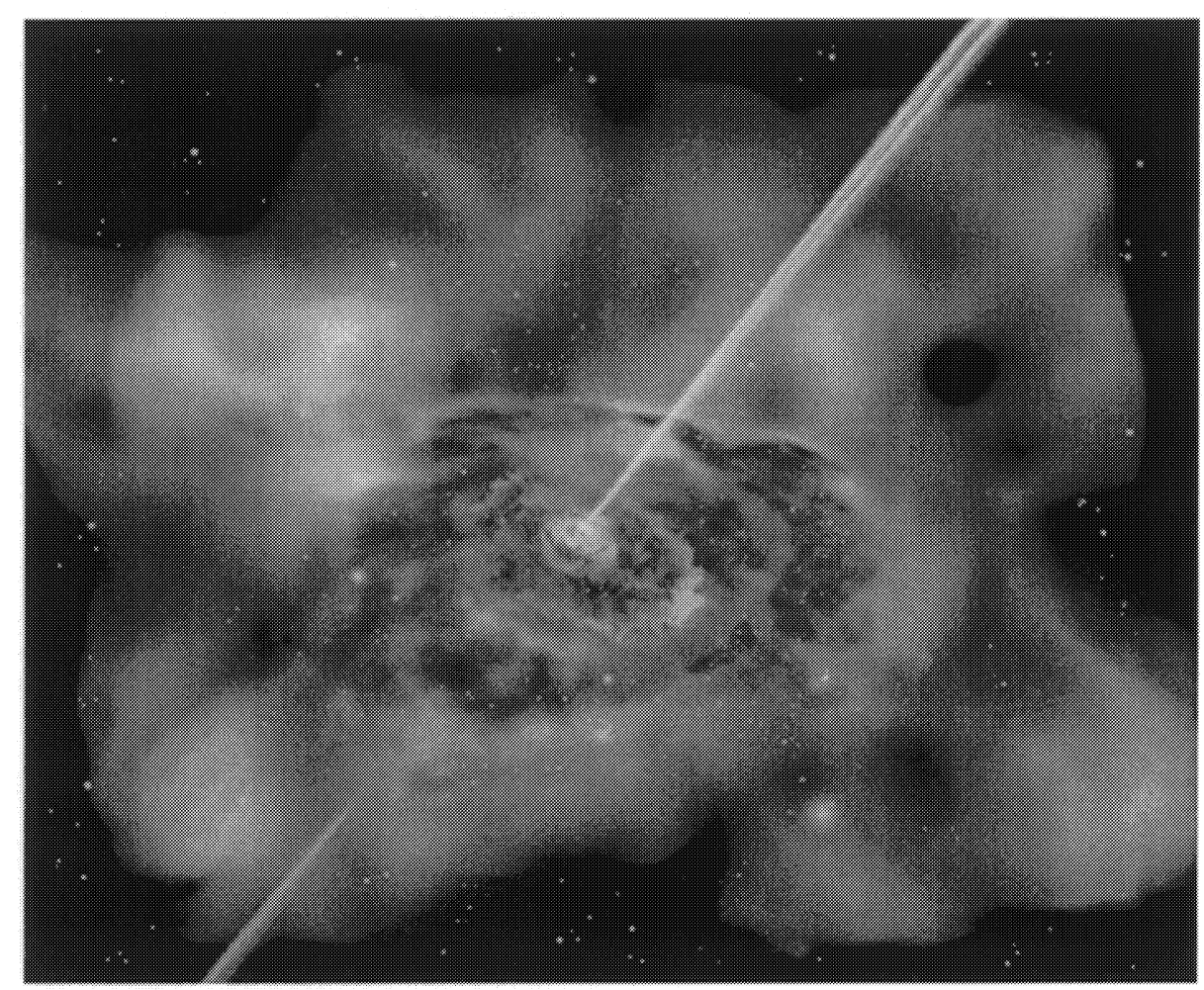

Figure 1. Artist's concept of The inner regions of an active galaxy, showing the dusty outer torus and the relativistic jets. (Figure courtesy of L. Cominsky, reprinted with permission).

Our SIM observing program minimizes systematic errors in the computed astrometric positions of targets, by relying primarily on observations within a SIM "tile" (a circular region $15^{\circ}$ in diameter). The specific SIM observations are:

1. We will measure the angular separation between (a) archetypal core-jet AGN, (b) quasars selected for their compactness from the radio sources that define the extragalactic reference frame, and (c) reference stars in four "target-rich" tiles, e.g., a 15-degree field of regard containing 3C 273 and M87. Comparison of the optical angular separation between archetypal AGN and extragalactic reference frame quasars with the radio angular separation measured with VLBI, over the five-year mission lifetime, enables us to assess the potential quality of a radio-optical frame tie.

2. We will use multicolor differential phase techniques to measure the size and stability of the optical continuum emission which will tell us how the optical photocenter location depends on wavelength.

3. We will determine quasar positions within a tile to $20 \mu$ as within one year, using monthly observations. The precision within the tile is independent of the full mission Grid Star Reference Frame. Significant early science results, and validation of the optical-radio frame tie, can be obtained during the first year of SIM operations.

In the following sections, we address each question in turn, and our method for answering it. We use $H_{o}=75$ $\mathrm{km} / \mathrm{s} / \mathrm{Mpc}$ and $q_{o}=0.5$. At the assumed $15-\mathrm{Mpc}$ distance of $\mathrm{M} 87,10 \mu \mathrm{as}=6.8 \times 10^{-4} \mathrm{pc}$; for a quasar at moderate redshift, such as $3 \mathrm{C} 345$ at $z=0.595,10 \mu$ as $=0.05 \mathrm{pc}$. 


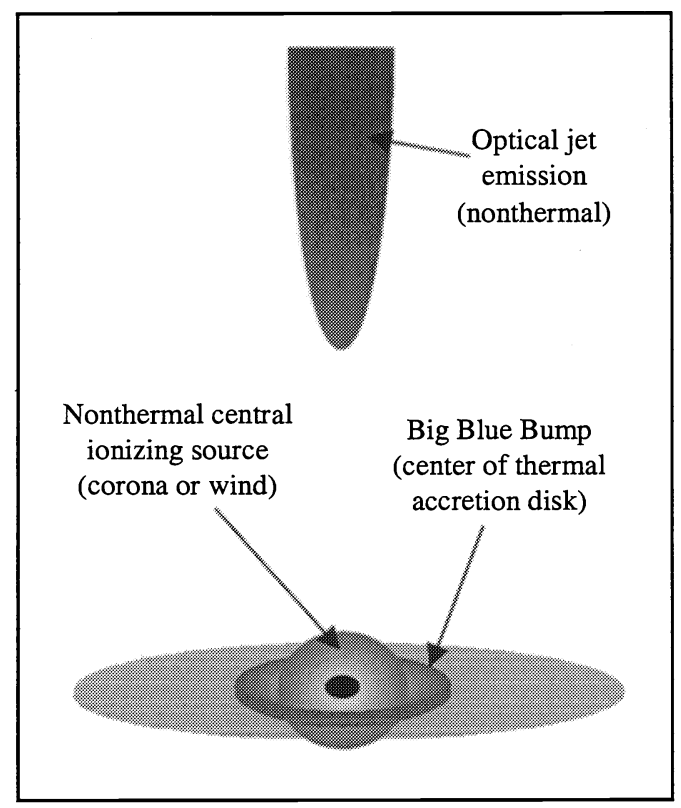

Figure 2. Model of the central quasar accretion disk (after Band \& Malkan 1989) and jet (after Königl 1981) for a typical AGN. The size of the central disk+corona is about $70-120 \mathrm{R}_{\mathrm{Sch}}(100-160 \mu$ as at a distance of $15 \mathrm{Mpc}$, or only $1-2 \mu$ as at a redshift of 0.5 ). The positional offset between the optical disk emission and jet for $3 C 345(z=0.595)$ is expected to be of order $0.4 \mathrm{pc}$ or $\sim 80 \mu$ as.

\section{SCIENCE PROGRAM}

\subsection{The Location of the Most Compact Optical Emission}

\subsubsection{The Accretion Disk and Hot Corona or Wind Components}

Because quasar optical emission is unresolved by ground telescopes, our understanding of the inner structure of quasars is based on theoretical models of this emission. Figure 2 shows one of the leading models. It contains an accretion disk that produces optical continuum emission in at least two places.

Thermal emission. For reasonably high accretion rates (within, say, $10 \%$ of the Eddington limit), much of the disk will be geometrically thin and optically thick to free-free continuum emission. This will produce a broad band of thermal emission which will peak in the near ultraviolet region. All objects with a bright optical central source (Seyfert galaxies, broad-line radio galaxies, and quasars) are believed to be in this accretion state, and many quasars display such a peak in their spectra - the "Big Blue Bump". Using disk models developed by Shakura \& Sunyaev, ${ }^{3}$ we find that the size of the part of the disk that produces this $10^{4} \mathrm{~K}$ emission will be $\sim 3.6 \times 10^{16} \mathrm{~cm}$, or $0.012 \mathrm{pc}$ for a typical $10^{9} M_{\odot}$ black hole, accreting at $10 \%$ of the Eddington rate. For M87, at a distance of $15 \mathrm{Mpc}$, this region would subtend an angular size of $\sim 160 \mu$ as. For a quasar at a redshift of $0.5(D \sim 1 \mathrm{Gpc})$, its angular size would be only $\sim 2 \mu$ as.

Non-thermal emission. Because it has a high frequency thermal cut-off, the Big Blue Bump will not be the most important source of optical photo-ionizing radiation in quasars, however. The main source appears to be a component producing a steep power-law spectrum that extends from the infrared into the far ultraviolet. (Only such a hard spectrum can produce the observed quasar emission lines of highly-ionized carbon, silicon, nitrogen and oxygen [Osterbrock \& Matthews $\left.{ }^{4}\right]$ ). Because of its power-law spectrum, this emission is probably nonthermal (either optical synchrotron or Comptonized emission from a lower-frequency radio or sub-millimeter source). While a beamed relativistic radio jet can produce such non-thermal optical emission, it is unlikely that $a$ jet is the primary source of this ionizing radiation in all quasars. One reason is that most quasars are 
radio quiet, indicating that their jets are very weak or non-existent. Furthermore, when such a jet does exist, its optical and radio emission will be beamed into a very narrow cone, affecting only a fraction of the gas surrounding the quasar. Models of quasar emission line regions, on the other hand, indicate that the ionizing radiation is consistent with being nearly isotropic. In fact, for some quasars, the broad line regions appear to fit a Keplerian (disk) profile, which is significantly different from a jet structure (Eracleous \& Halpern ${ }^{5}$ ). One possible physical model for the central ionizing source is a magnetized corona or wind ejected from the central region of the accretion disk. Such a structure would emit infrared/optical/ultraviolet synchrotron as well as thermal and Comptonized X-rays. Models of this region (e.g., Band \& Malkan ${ }^{6}$ ) indicate a size of only $\sim 70$ Schwarzschild radii, centered on the black hole even at microarcsecond resolution. At a redshift of $z=0.5$, it would have an angular size of only $\sim 1 \mu$ as.

To summarize, for AGN in which the disk emission dominates (radio quiet objects with little or no jet, or radio loud objects viewed at a large angle to the jet axis), we expect that emission in the red region of the spectrum (dominated by the non-thermal ionizing source) will be positionally coincident with the blue emission (dominated by the thermal disk) at the microarcsecond level. Both regions of emission will be physically centered on the black hole. This also will be true for objects without a strong blue bump (completely dominated by the central ionizing source). Any color-dependent position shift in such AGN seen with SIM observations would indicate additional structure in the object that is not predicted by the standard quasar models.

\subsubsection{The Beamed Relativistic Jet}

About $10 \%$ of AGN possess a powerful relativistic jet. Figure 3 shows a montage of the parsec-scale radio jet of $3 \mathrm{C} \mathrm{279}$; the compact blobs in the jet in this image extend roughly 4 milliarcseconds and move at $0.997 \mathrm{c}$ (Wehrle et al. ${ }^{7}$ and Piner et al. $\left.{ }^{8}\right)$. Jets appears to be ejected in a direction approximately perpendicular to the accretion disk (Figures 1 and 2). The process of acceleration and collimation of jets appears to take place near the central regions of a magnetized accretion disk, with centrifugal and magnetic pressure forces driving the outflow and magnetic pinch forces effecting the collimation. However, as the rate of occurrence of powerful jets in AGN is small, additional factors are believed to be important. In particular, it has been proposed that a powerful jet cannot be produced unless the central black hole is spinning rapidly (Wilson \& Colbert ${ }^{9}$; Blandford ${ }^{10}$; Meier ${ }^{11}$ ).

If an AGN is radio-loud, and viewed at a small angle to the jet axis (a "blazar" like 3C 279 or 3C345), then there is a third possible compact source of optical continuum emission in the system which would be detectable by SIM: emission from the relativistic jet beamed toward the observer. The radio emission in such objects is also very compact. The Königl model for relativistic jets (Königl ${ }^{12}$ ) predicts that, in the optical, the majority of the emission comes not from synchrotron emission from the central portion of the jet but rather from synchroself-Compton emission in the region of the jet where the synchrotron emission peaks in the radio or millimeter (Hutter \& Mufson $\left.{ }^{13}\right)$. A detailed application of this model to 3C $345(z=0.595)$, for example, predicts the optical emission to lie $\sim 80 \mu$ as from the other two above emission regions (i.e., from the black hole) nearly coincident with the $22 \mathrm{GHz}$ radio emission ( $\sim 70 \mu$ as or $0.4 \mathrm{pc}$ from the black hole). SIM can test the Königl model predictions directly.

\subsubsection{Distinguishing AGN Models using Color-Dependent Astrometry}

We will use SIM's ability to precisely determine changes in position between red and blue light from a single source to test two general models for the optical structure of AGN, as outlined above:

Model 1 Most of the red light is power-law synchrotron emission along the relativistic jet.

Model 2 Red light comes from synchrotron or inverse Compton emission from a hot, magnetized corona or wind above the accretion disk.

In both models, most of the blue light is thermal emission from the optically thick part of the accretion disk the source of the "Big Blue Bump" in many quasars and AGN. The discriminator between these is is very simple: is there an offset between red and blue photocenters? See Figure 2 for a sketch of the AGN emission regions on the size scale relevant for SIM. The models have the following observational consequences: 


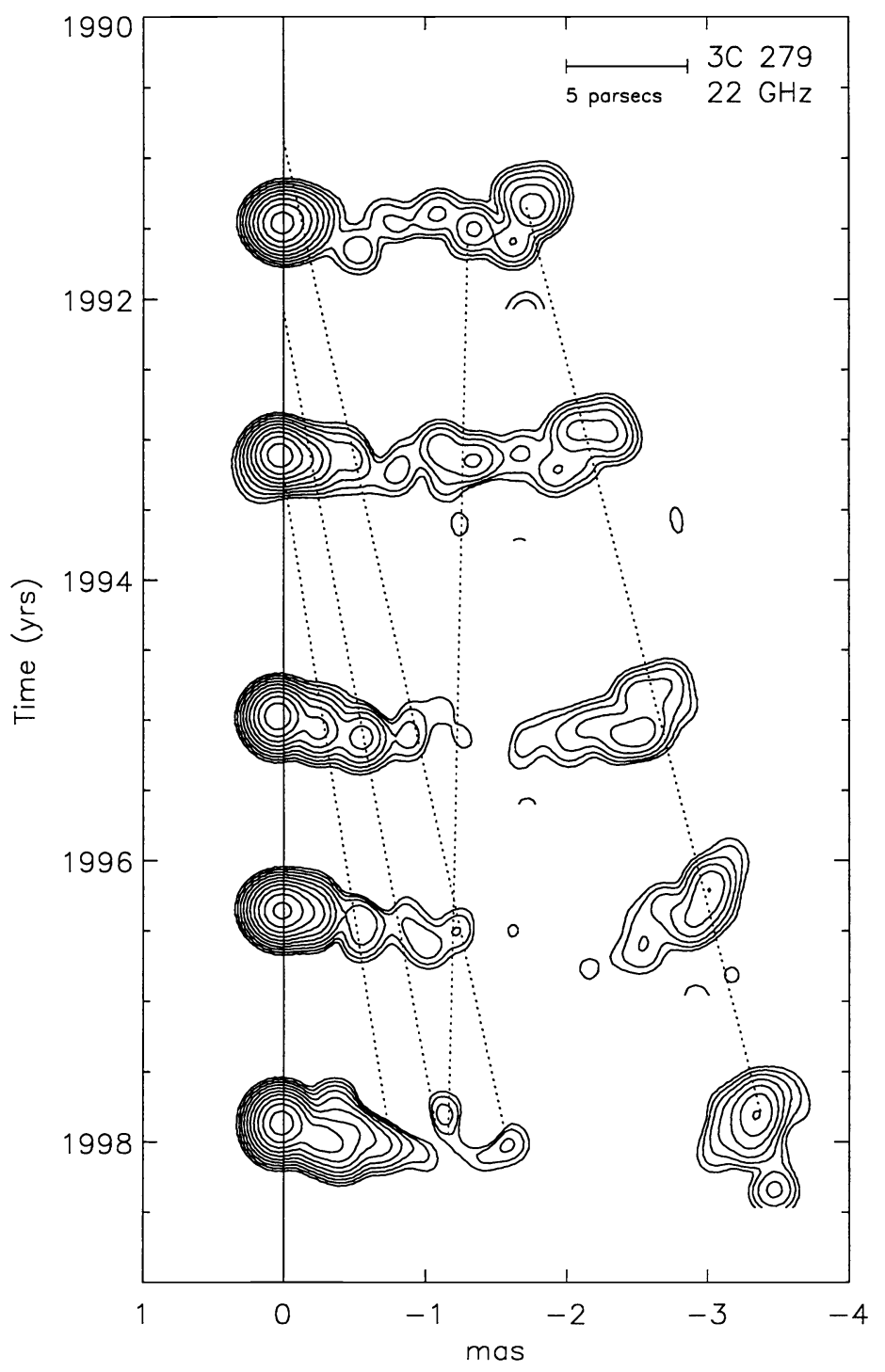

Figure 3. Mosaic of radio images made with the NRAO VLBA at $22 \mathrm{GHz}$, showing superluminal motion of radio blobs (right) away from the core (left). See Wehrle et al. 2000 for details.

In Model 1, red light comes from the jet, so its photocenter will be offset (along the position angle of the jet) from the blue photocenter associated with the accretion disk. In Model 2, red light comes from a disk corona or wind, so the red and blue photocenters should be coincident.

We can measure the offset between red and blue photocenters, and monitor any changes in the separation, by measuring the phase shift of the white light fringe between the red and blue halves of the SIM detector. This is a differential measurement, and is largely independent of the absolute delay accuracy or precise knowledge of baseline orientation, and depends only on the available SNR of the detected fringes. In addition, by repeating the tile observations during the mission, we can learn whether this location is stable over time.

\section{Examples of What SIM Should See in Specific Targets}


M87: In this nearby radio galaxy, we expect the optical emission to be dominated by the accretion disk region because its jets are not pointing within a few degrees to our line of sight. We therefore expect relatively large offsets between the optical photocenter and radio photocenter (which is dominated by emission from the optically thick base of the radio jet). As noted below, this shift is expected to be on the order of $100 \mu$ as for 3C 345, and should be even larger for this low-redshift radio galaxy where the angular-linear scale is larger and the radio jets are not seen in projection.

3C 345 and MKN 501: In 3C 345 (Unwin et al. ${ }^{14}$ ), optical jet emission should be roughly coincident with the $22 \mathrm{GHz}$ radio emission, at about $80 \mu$ as from the black hole. Color-dependent position shifts may be observable across the SIM band analogous to the frequency-dependent separation effect well-known in VLBI observations (the Königl model predicts a shift of $30 \mu$ as from the red to the blue end of the SIM band for 3C 345). In the TeV blazar MKN 501, the maximum synchrotron turnover frequency does not occur until the optical band or higher. in this case we expect the optical synchrotron emission to come from the base of the jet very close to the black hole and well-separated from the radio emission further out.

\subsection{The Radio-Optical Frame Tie}

Does the separation of the radio core and optical photocenter of the quasars used for the reference frame tie change on the timescales of their photometric variability, or is the separation stable? The radio sources used in the International Celestial Reference Frame are the most compact sources known. The most compact, flat-spectrum sources are also usually the most variable at radio and optical wavelengths. Radio flux variability is correlated with changes in structure on milliarcsecond scales, as we know from studying superluminal sources with VLBI. We observe optical jets in at least two of our target sources (M87 and 3C273), yet we do not have any optical images with resolution comparable to VLBI. Perlman et al. ${ }^{15}$ have shown how polarized optical and radio features in M87 ("knots") on fraction-of-an-arcsecond scales (using Hubble Telescope and NRAO VLA data) are correlated. We therefore think there is a significant possibility that the ICRF quasars may show shifts in their optical centroids; observed optical photometric variability has timescales of minutes to months. While our group is collaborating with our colleagues Johnston et al. (at USNO; see their paper ${ }^{16}$ in these proceedings) to identify the optimal ICRF quasars for the frame tie, we are fully aware that it will not be possible to guarantee that the objects are unresolved on the important SIM angular scales. The topics most affected by the accuracy of the reference frame tie are Galactic structure and extragalactic rotational parallaxes.

By devoting intense, early study to the nearest quasar and to archetypal quasars, as well as those believed to be oriented directly towards us, in the first year of the SIM mission, we should get a solid handle on the extent to which the optical photocenters are (a) well-defined (b) compact and (c) moving or stationary. The nearest quasar, 3C 273, will certainly not be a frame tie object because of its extended radio jet, but its proximity allows us to study variability with minimal effects of time-dilation. We will measure the angular separation between our scientifically interesting quasars and our ICRF quasars with both SIM and ground-based VLBI, and directly compare the radio and optical separations.

\subsection{Finding Binary Black Holes With SIM}

What happens to the supermassive black holes at the centers of galaxies, when two galaxies collide and coalesce? If mergers are in fact common, then knowing the fate of their central regions is central to understanding how non-thermal activity in galactic nuclei begins, and how it evolves with time. If massive binary black holes are found, we will have a new means of directly measuring their masses and estimating the coalescence lifetimes of the binaries.

Near the end of a galactic merger, a system comprising a black hole and an accretion disk system may find itself in a close orbit about a similar system (Figure 4). The time scales for the nuclei to merge, and the black holes to form a binary, are typically a few hundred million years - short compared to the Hubble time. The binary phase can last a similar amount of time until the black hole separation much less than one parsec, at which time gravitational radiation losses cause it to coalesce more rapidly. The gravitational radiation lifetime is about $3 \times 10^{6} \mathrm{yr}\left(\mathrm{Krolik}^{17}\right)$. The frequency of occurrence of binary black holes in the general quasar population is expected to be small, hence, we will select the best candidates (such as OJ 287; see Kidger ${ }^{18}$ ) during our pre-launch Preparatory Science Program. 


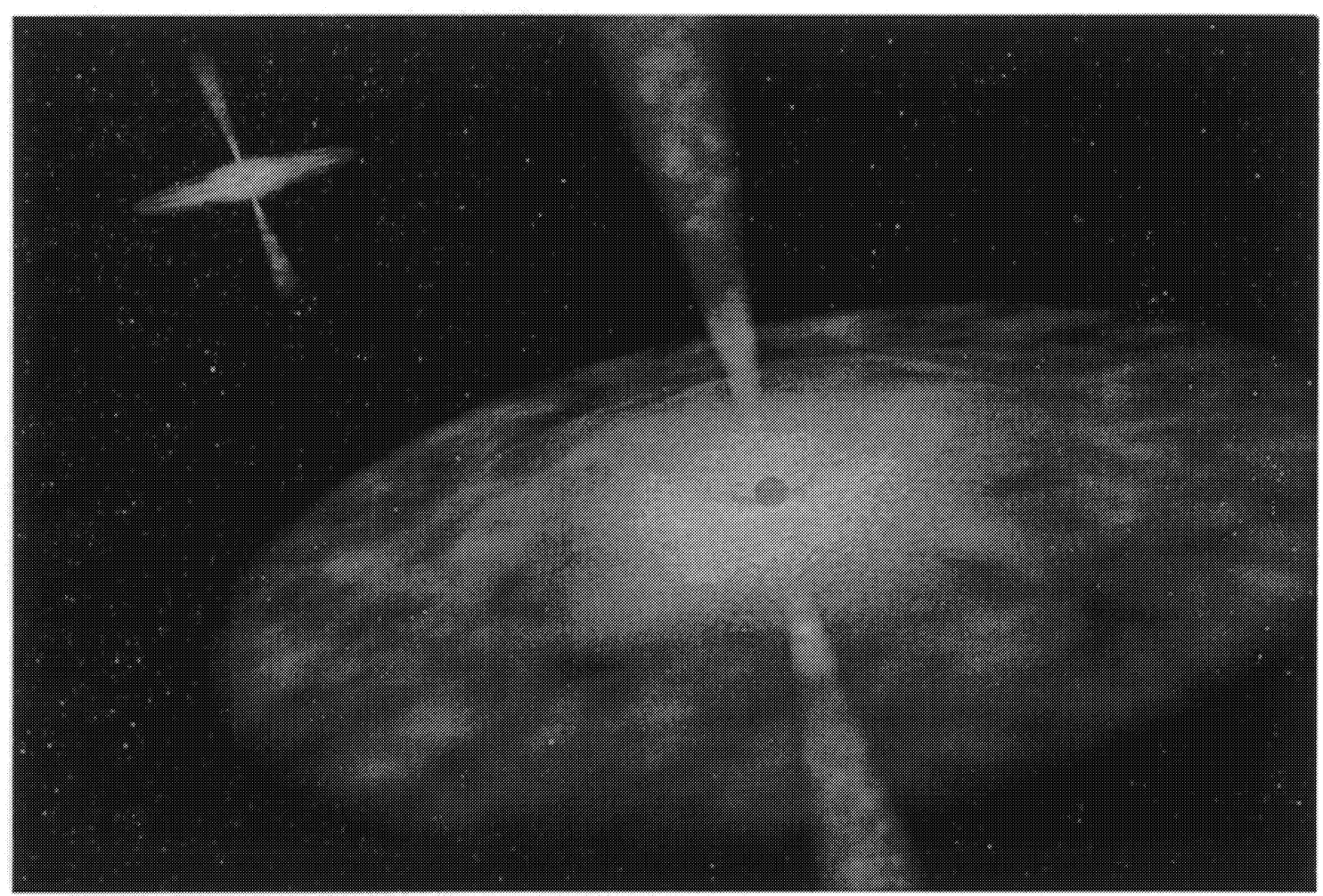

Figure 4. Schematic diagram of a possible binary black hole system. Such a system could have a number of different configurations. The emission could be tied to either the more massive hole or its companion.

We can detect binary black holes by measuring optical position changes due to orbital motion, just as SIM can detect periodic shifts in the photocenters of stars caused by much smaller orbiting planets. The very best AGN to examine for binary black holes in their cores are those whose optical light is dominated by beamed emission from the relativistic jet rather than thermal emission from the accretion disk. The reason for this is that beamed jet emission is expected to be associated with an individual black hole in a binary system, while thermal disk emission may come from a region surrounding both black holes (a "shared" accretion disk whose scale is larger than the black hole separation). Thus, we plan to select primarily those objects whose jets are pointed directly at us: the blazer class we have been studying for many years, and which make up the vast majority of the International Celestial Reference Frame (ICRF) sources (see, for example, Unwin et al., ${ }^{14}$ Wehrle et al., ${ }^{19}$ Piner \& Kingham ${ }^{20}$ ).

In summary, the test for binary black holes is to observe the periodic astrometric reflex motion ("wobble") of the photocenter of a blazar over the course of the SIM mission. A binary will trace an elliptical path. If the detected motion is random, then the blazar shows no evidence of binarity. Any detection of astrometric motion will be a major discovery for SIM, providing a fundamentally different kind of measurement with which to confront theories of active galaxy structure and emission.

\section{OBSERVING PROGRAM}

The observational goals of our AGN program require: (1) Relative positions and proper motions between AGN of several different classes; and (2) Color-dependent position offsets using differential phase information on individual AGN.

We can achieve all of these goals by limiting our target selection to those which "fit" within a small number of SIM tiles,. A sample tile is shown in Figure 5. Since our science objectives can be met using relative measurements between AGN, we place only limited reliance on the astrometric grid constructed by the SIM 


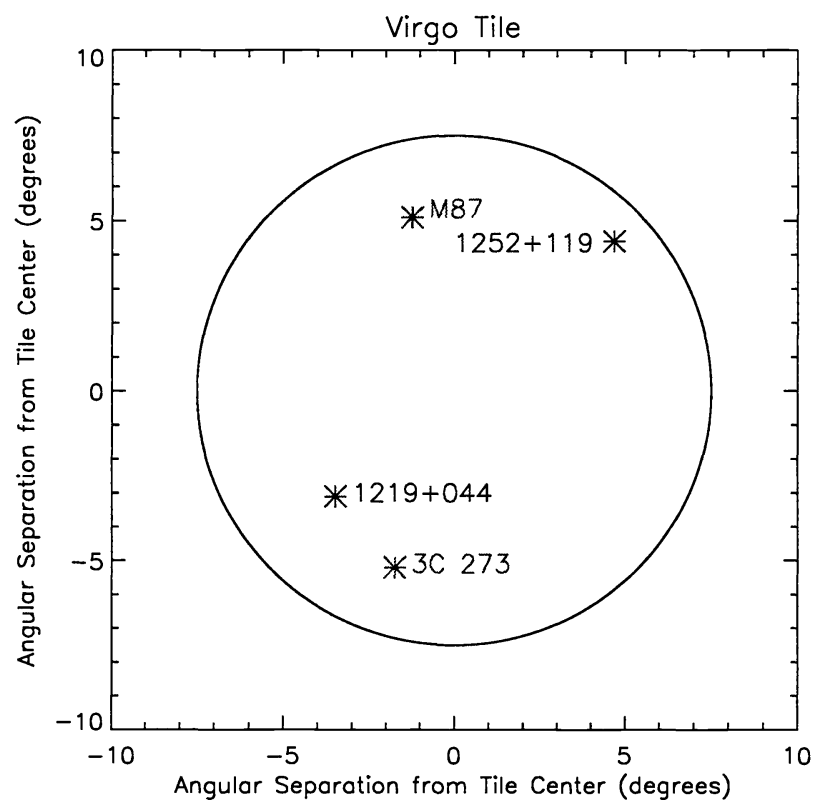

Figure 5. Sample tile in the Virgo region. Prime science targets are M87 and 3C273. Nearby compact ICRF quasars are $1252+119$ and $1219+044$.

Project. Thus our observing plan does not require global astrometry; instead we perform local astrometry within a single tile. And because we do not depend on the grid, we can obtain results in the first year, when the anticipated grid accuracy will be much poorer than at end-of-mission.

By concentrating the observations within a tile, we gain over the standard wide-angle observation scenario, both in terms of reduced observing overhead, and improved instrument performance. While the selected AGN are too faint to enable the rapid-switching which will be used for planet-searching, we avoid picking up astrometric errors from the grid itself.

Each tile will be observed in a time-efficient manner which shares characteristics of both standard narrowand wide-angle SIM modes. That is, we observe grid stars to determine the basic instrumental parameters, followed by the science targets. Depending on the instrument stability over $\sim$ hour timescales, we may repeat the grid star observations at the end of a tile, to eliminate first-order drifts. AGN positions will be computed in the coordinate frame of the grid, and refined as the grid improves during the mission. But the science will be derived primarily from differences in $\sigma_{\mathrm{AGN}}$ for each target at the epoch in question. Because most of our targets are relatively faint, our data will be dominated by photon noise, rather than instrument systematic errors.

\section{SUMMARY}

SIM offers unique opportunities for the study of active galactic nuclei. Quasar observations with SIM will anchor its astrometric reference grid, thereby forming a reference system 25 times more accurate than the current ICRF standard. Astrometry with SIM is a powerful new tool for learning about AGN phenomena: relativistic jets, hot coronae, accretion disks, and supermassive binary black holes. Astrometry is a fundamentally new type of observing for AGN, allowing the study of phenomena on size scales much smaller than the resolution of the best imaging telescopes, both in space and on the ground. The current state of the art in optical astrometry is $\sim 500 \mu$ as in space, and much poorer from the ground, whereas SIM will detect the astrometric shift in the photocenter position at $10 \mu$ as or less. Optical astrometric shifts have yet to be seeen in any extragalactic object; however, we have shown that the prospects for exciting discoveries with SIM are excellent. SIM astrometric data on AGN will be combined with measurements made with more traditional methods, such as optical imaging, spectroscopy, photometry and VLBI radio imaging, to yield new insights into the most powerful objects in the universe. 


\section{ACKNOWLEDGMENTS}

The research described in this paper was carried out at the Jet Propulsion Laboratory, California Institute of

Technology, under a contract with the National Aeronautics and Space Administration.

\section{REFERENCES}

1. L. Ferrarese, H.C. Ford, and W. Jaffe "Evidence for a Massive Black Hole in the Active Galaxy NGC 4261 from Hubble Space Telescope Images and Spectra", Ap.J., 470, 4441996.

2. W. Jaffe, H. Ford, L. Ferrarese, F. van den Bosch, R.W. O'Colnnell, "The Nuclear Disk of NGC 4261: Hubble Space Telescope Images and Ground-based Spectra", Ap.J., 460, 214, 1996.

3. N. I. Shakura \& R.A. Sunyaev, "Black holes in binary systems. Observational appearance" A. $8 A$. 24, 337, 1973.

4. D. E. Osterbrock \& W. G. Mathews, "Emission-Line Regions of Active Galaxies and QSOs", A.R.A.A., 24, 171-203, 1986.

5. M. Eracleous, \& J.P. Halpern, "Doubled-peaked emission lines in active galactic nuclei" Ap.J.S., 90, 1, 1994.

6. D. L. Band, \& M.A. Malkan, "Synthesis of accretion disk and nonthermal source models for active galactic nuclei" Ap.J., 345, 122, 1989.

7. A. E. Wehrle, B.G. Piner, S. C. Unwin et al. "Kinematics of the Parsec-Scale Relativistic Jet in Quasar 3C 279: 1991-1997" Ap.J.S., 133, 297, 2001.

8. B. G. Piner, S. C. Unwin, A. E. Wehrle, A.C. Zook, C. M. Urry, \& D. M. Gilmore, "The Speed and Orientation of the Parsec-Scale Jet in 3C 279", to be submitted to Ap.J. in August 2002.

9. A. S. Wilson \& E. J. M. Colbert, "The difference between radio-loud and radio-quiet active galaxies", Ap.J., 438, 62, 1995.

10. R. D. Blandford, "Relativistic Accretion" in Astrophysical Disks, Proceedings of ASP Conf. Ser., Vol. 160, ed. J. A. Sellwood and J. J. Goodman, 265, 1999.

11. D. L. Meier, "Grand unification of AGN and the accretion and spin paradigms" New Astronomy Reviews, 46, 247, 2002.

12. A. Königl, "Relativistic jets as X-ray and gamma-ray sources", Ap.J. 243, 700. 1981.

13. D. J. Hutter \& S. L. Mufson, "Application of a relativistic jet model to the study of BL Lacertae objects" Ap.J., 301, 50, 1986.

14. S.C. Unwin, A.E. Wehrle, C.M. Urry, D. M. Gilmore, E. J. Barton, B. C. Kjerulf, J. A. Zensus, \& C. R. Rabaca, "Inverse Compton X-ray emission from the superluminal quasar 3C 345" ApJ, 432, 103. 1994.

15. E.S. Perlman, J.A. Biretta, F. Zhou, W. B. Sparks, F. D. Macchetto, "Optical and Radio Polarimetry of the M87 Jet at 0.2" Resolution" A.J., 117, 2185, 1999.

16. K. J. Johnston, A. Fey, D. Boboltz, R. Gaume, N. Zacharias, "Astrophysics of Reference Frame Tie Objects", SPIE Paper 4852-15, 2002.

17. J. Krolik, Active Galactic Nuclei, Princeton University Press, Princeton, 1999.

18. M. Kidger, "The 11 Year Period in OJ 287 Revisited: Is It a True Long-Enduring Period?" $A J$, 119, 2053, 2000.

19. A. E. Wehrle, E. Pian, C. M. Urry et al. "Multiwavelength Observations of a Dramatic High-Energy Flare in the Blazar 3C 279", ApJ 497, 178, 1998.

20. B. G. Piner \& K. A. Kingham, "Geodetic VLBI Observations of EGRET Blazars" Ap.J. 507, 706, 1998. 